\title{
Perfil epidemiológico dos casos de morte materna na cidade de Manaus por: causa, escolaridade e raça, no período de 2011 a 2015
}

\author{
Epidemiological profile of maternal death cases in the city of Manaus by: cause, class, \\ schooling and race, in the period of 2011 to 2015
}

Perfil epidemiológico de los casos de muerte materna en la ciudad de Manaus por: causa, clase, escolaridad y raza, en el período de 2011 a 2015

Alexsandro Sampaio de Oliveira ${ }^{1 *}$, Dayson José Jardim Lima², Nadionara Costa Menezes ${ }^{3}$.

\section{RESUMO}

Objetivo: Traçar o perfil epidemiológico da morte materna em Manaus, criando indicadores de sensibilidade. Métodos: Trata-se de um estudo ecológico com finalidade quantitativa, com base na análise de dados secundários dos serviços de informações humanas governamentais como da própria Secretária Municipal de Saúde, utilizando para isto a experimentação randomizada num período compreendido entre 2011 a 2015, baseando-se na prevalência da saúde da população feminina, a presente pesquisa identificou grupos populacionais em risco de morte materna. Resultados: Nos anos de 2011 a 2015, registrou-se 136 casos de Morte Materna, permitindo-nos estabelecer indicadores de sensibilidade, quanto frequência e condição socioeconômica, estratificando-os da seguinte forma: Nível Educacional entre 8 e 11 anos estudados, com 38\%; Prevalência Racial de $79 \%$ entre mulheres parda; as Mortes Maternas Obstétricas Diretas estão ocupando $55 \%$ dos casos, dividindo-se entre as doenças conhecidas como; Doença Específica da Gravidez e Infecção Puerperal. Conclusão: Conclui-se que a maioria da Mortes Maternas ocorreram por causas obstétricas diretas, ou seja, causas evitáveis podendo ser prevenidas com uma atenção de qualidade em todos os níveis de assistência à saúde. Observa-se a disparidade entre o número de gestantes na Faixa etária de 20 a 29 e Escolaridade entre 8 e 11 anos.

Palavras-chave: Mortalidade, Indicadores de sensibilidade, Perfil epidemiológico.

\begin{abstract}
Objective: To trace the epidemiological profile of maternal death in Manaus, creating sensitivity indicators. Methods: This is an ecological study with a quantitative objective, based on the analysis of secondary data from the governmental information services, such as the Municipal Health Secretary, using randomized trial in a period between 2011 to 2015 , based on the prevalence of female population health, the present study identified population groups at risk of maternal death. Results: In the years 2011 to 2015, 136 cases of maternal death were recorded, allowing us to establish sensitivity indicators, frequency and socioeconomic status, stratifying them as follows: Education Level between 8 and 11 years studied, with $38 \%$; Racial Prevalence of $79 \%$ among brown women; Direct Obstetric Maternal Deaths are occupying $55 \%$ of the cases, dividing among the diseases known as; Specific Disease of Pregnancy and Puerperal Infection. Conclusion: It is concluded that most of the Maternal Deaths occurred due to direct obstetric causes, that is, preventable causes and can be prevented with a quality care at all levels of health care. It is observed the disparity between the number of pregnant women in the age group of 20 to 29 and Schooling between 8 and 11 years.
\end{abstract}

Keywords: Mortality, Maternal Death, Sensitivity Indicator, Epidemiological Profile.

${ }^{1}$ SOEPEC Sociedade de Ensino Profissionalizante Educacional e Cultural, Teresina - PI.

*E-mail: alexsandro.s.o@hotmail.com

${ }^{2}$ Centro Universitário Luterano de Manaus - (CEULM/ ULBRA), Manaus - AM.

${ }^{3}$ Secretaria Municipal de Saúde - (SEMSA), Manaus - AM.

SUBMETIDO EM: $2 / 2019$ 


\section{RESUMEN}

Objetivo: Trazar el perfil epidemiológico de la muerte materna en Manaos, creando indicadores de sensibilidad. Métodos: Se trata de un estudio ecológico con finalidad cuantitativa, con base en el análisis de datos secundarios de los servicios de informaciones humanas gubernamentales como de la propia Secretaria Municipal de Salud, utilizando para ello la experimentación aleatorizada en un período comprendido entre 2011 a 2015, en la prevalencia de la salud de la población femenina, la presente investigación identificó grupos poblacionales en riesgo de muerte materna. Resultados: En los años 2011 a 2015, se registraron 136 casos de Muerte Materna, permitiéndonos establecer indicadores de sensibilidad, como frecuencia y condición socioeconómica, estratificándolos de la siguiente forma: Nivel Educativo entre 8 y 11 años estudiados, con 38 $\%$; Prevalencia Racial del $79 \%$ entre mujeres parda; las muertes maternas obstétricas directas están ocupando el $55 \%$ de los casos, dividiéndose entre las enfermedades conocidas como; Enfermedad Específica del Embarazo e Infección Puerperal. Conclusión: Se concluye que la mayoría de las muertes maternas ocurrieron por causas obstétricas directas, o sea, causas evitables pudiendo ser prevenidas con una atención de calidad en todos los niveles de asistencia a la salud. Se observa la disparidad entre el número de gestantes en la franja de edad de 20 a 29 y la escolaridad entre 8 y 11 años.

Palabras clave: Mortalidad, Indicadores de sensibilidad, Perfil epidemiológico.

\section{INTRODUÇÃO}

A Morte Materna (MM) traz em seu conceito, o óbito da mulher durante o período gestacional, até 42 dias após o seu término, independentemente da duração ou localização da gravidez, devida a qualquer causa relacionada e agravada pelo estado gestacional, ou por medidas tomadas em relação a ela, porém não devida a causas acidentais ou incidentais (VIANA JFS, 2004).

Morte materna obstétrica direta (MMOD), aquela resultante de complicações obstétricas, durante o estado gestacional, parto ou puerpério devidas em intervenções, omissões, tratamentos incorretos ou de uma sequência de eventos resultantes de qualquer uma dessas situações; e a Morte materna obstétrica indireta (MMOI), é aquela que resulta de doença preexistente ou que se agrave ou desenvolve durante o período gestacional, não devida a causas diretas, mas agravada pelos efeitos fisiológicos da gravidez (ONUBR, 2016).

A classificação da MM é definida em duas causas distintas a primeira em Morte Materna Obstétrica Direta (MMOD), que são complicações obstétricas no período gravídico, no parto ou puerpério e consequentes de doenças como; a Doença Hipertensiva Especifica da Gravidez (DHEG), Infecção Puerperal, anomalias gestacionais (gravidez ectópicas), bem como intervenções, omissões, tratamento incorreto ou ocorrência concomitante de qualquer uma dessas. A segunda se conceitua pela Morte Materna Obstétrica Indireta (MMOI) que resultada de doenças pregressas a gestação, como; doenças cardíacas, renais, insuficiência respiratória e virais, ou que se desenvolva neste período, agravando-se pelos efeitos fisiológicos da gravidez (SOUZA KV et al, 2011). A Organização das Nações Unidas do Brasil (ONUBR), classifica a Razão de Mortalidade Materna (RMM) do seguinte modo: RMM baixa, quando menor que 20 óbitos por 100.000 Nascidos Vivos (NV), RMM alta é classificada com valores entre 50 e 149 óbitos maternos e RMM muito alta a partir de 150 por $100.000 \mathrm{NV}$ (BRASIL, 2012).

Estudo sobre a RMM realizado pelo Ministério da Saúde (MS) e Serviço de Vigilância em Saúde (SVS), aponta que a RMM passou de 143 óbitos por 100 mil NV, em 1990, para 70 óbitos por 100 mil NV no ano de 2010. Como principais causas MM, nos anos de 2000 a 2009 foram relacionadas: outras doenças obstétricas diretas que complicaram na gravidez, parto e puerpério (17,10\%); eclampsia $(11,88 \%)$; hipertensão gestacional com proteinúria elevada $(6,22 \%)$; hemorragia pós-parto $(5,86 \%)$; infecção puerperal $(5,18 \%)$ e descolamento prematuro de placenta $(4,28 \%)$. No Brasil, em 2014, foram registrados $1.552 \mathrm{MM}$, por 100 mil NV, com maior incidência nas regiões do Sudeste, com 540 MM, por 100 mil NV (BRASIL, 2015; MARTINS, SILVA, 2015).

A região norte do Brasil é a segunda área de maior prevalência de morte maternos e os locais de maior incidência são as maternidades públicas. O período de 2001 a 2010, houveram 6392 casos de morte de em 
mulheres na idade fértil em Manaus, desses casos, 241 foram classificados como MM, resultando em uma RMM de 63,2/100 $000 \mathrm{NV}$. As MMOD representaram 162 casos (67,2\%), enquanto as MMOI caracterizaram 62 casos (25,7\%). As Morte Maternas Obstétricas Não Especificadas (MMNE) constituíram 17 casos (RAMOS SCS, 2013; CASTRO BMC, RAMOS SCS, 2016).

Tratando-se de Manaus, nos anos de 2011 a 2015 foram registrados pela SEMSA 212.585 NV e 135 casos de MM, dentre esses em seu acumulado podemos traçar como indicadores de sensibilidade os grupos mais atingidos, são eles; as Mulheres com escolaridade entre 8 a 11 anos de estudo e mulheres classificadas como pardas. As Morte Maternas Obstétricas Diretas (MMOD) estão ocupando o ápice dos casos. Os casos de MM que ocorrem no puerpério possuem pouca expressão com pouca representação de eventos no período tardio (SEMSA-MN, 2016).

Muitos casos de MM podem ser prevenidos com simples intervenções como: planejamento familiar, melhor atendimento pré-natal, atenção adequada durante o trabalho de parto e no período pós-parto, essas medidas podem reverter o quadro de MM. Nesta perspectiva o acompanhamento gestacional é imprescindível para uma gestação saudável, ao parto seguro e consequentemente redução da mortalidade. A fim de exercer melhor controle sobre a RMM, o Ministério da Saúde (MS) determinou que os centros de saúde realizassem as notificações de MM através de suas Secretarias Estaduais e Municipais de Saúde e os dados referentes ao binômio mãe e filho fossem enviados aos Comitês Municipais de Prevenção do Óbito Materno e Infantil (CMPOMIF), em seguida inseridos a plataforma de acompanhamento do SUS (BRASIL, 2012; CASTRO BMC, RAMOS SCS, 2016, WINGERTER DG, 2012).

Apesar da grande relevância dos estudos sobre MM em nosso país, ainda são escassos a forma estratificada do estudo, há muito para se conhecer, especialmente em certas regiões do país. Assim, o objetivo do estudo foi revisar pesquisas anteriores relacionadas ao tema e avaliar os dados ecológicos dos sites governamentais e cedidos pela Secretaria Municipal de Saúde (SEMSA), a fim de criar marcadores que identifiquem os grupos de risco e traçar o perfil epidemiológico da morte materna em Manaus, criando indicadores de sensibilidade para isso.

\section{MÉTODOS}

A pesquisa tratou-se de um estudo ecológico com finalidades quantitativa, realizado de forma exploratória, que utilizou para suas análises, as bases de dados secundários dos serviços governamentais de informações humanas, como: DATASUS, SIM, SINASC e SAGE, além de dados fornecidos pela própria Secretária Municipal de Saúde de Manaus - SEMSA-MN, que aceitou o projeto por apreciação autorizando-o através da Gerência de Gestão da Educação e Saúde, em janeiro de 2017, por parte dos profissionais avaliadores, que autorizaram o fornecimento de dados ecológicos referentes as Declarações de Óbitos (DO) e fichas de investigação de morte materna da cidade de Manaus, destes avaliou-se informações sociais e epidemiológicas das mortes maternas, como: causa, escolaridade e raça, os dados foram coletados, estratificados e interpretados de acordo com os preceitos legais vigente no país e de associações internacionais que lutam para a redução da MM.

A avaliação da pesquisa acompanhou o entendimento da comunidade científica nacional e internacional que vem descrevendo e compreendendo o fenômeno estatístico da Mortalidade Materna como um problema social e de saúde pública, para fins comparativos, utilizou-se também artigos científicos de revistas conceituadas, cuja as pesquisas realizaram-se nas bases de dados LILACS, pela Biblioteca Virtual de Saúde (BVS); MEDLINE, via PubMed, Scielo entre outras, que trataram o tema da morte materna em suas diversas variáveis ao longo dos anos. A fim de analisar a prevalência da saúde da população feminina gestante ou no período puerperal da cidade de Manaus, utilizou-se de dados já disponíveis e eticamente aceitável, o estudo apurou e quantificou dados em um determinado período de tempo, compreendido entre janeiro de 2011 a dezembro de 2015, baseando-se na prevalência da saúde da população feminina (Figura 1), a presente pesquisa identificou grupos populacionais em risco de morte materna, para comprovação deste foram confeccionados, tabelas e gráficos afim de nortear os resultados das amostras sobre Morte Materna e suas principais causas de forma estratificada, sistemática e imparcial (PRESTES, 2008). 
Figura 1 - Esquema representativo das pesquisas.

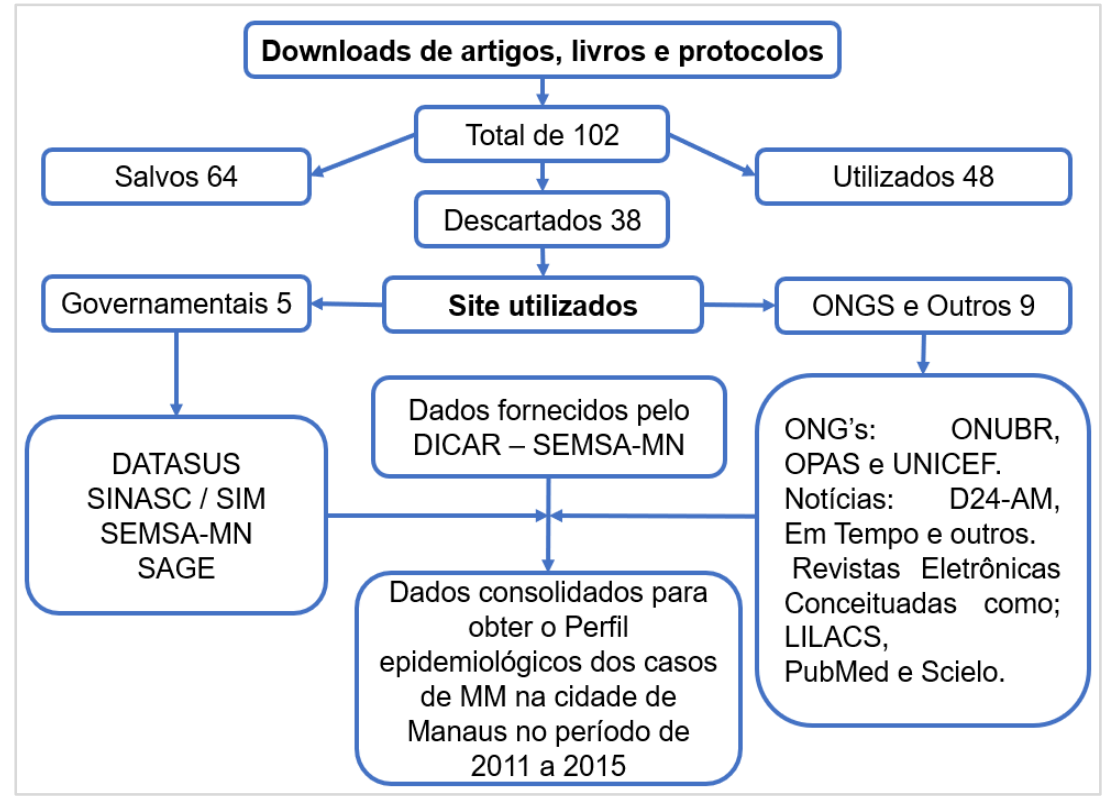

Fonte: SESMA, 2017.

\section{RESULTADOS}

A Morte Materna é um indicador que reflete a qualidade do sistema de saúde como; o acesso aos serviços, a qualidade da atenção básica, a educação em saúde e a assistência especializada nos hospitais. Observamos através de nosso estudo e colocamos a divisão dos casos mostrados em números naturais e estratificados na Tabela 1 abaixo, porém neste período as variações da classe de MM dividiu-se entre MMOI, MMOD e MMNE, respectivamente a MMOI apresentou 50 casos, cerca de $38 \%$, a MMOD teve 72 casos totalizando $55 \%$ e a MMNE possuiu apenas 10 casos, detendo apenas $8 \%$ do total das MM em Manaus.

Tratando-se da MMOD os casos foram muito diversificados entre suas principais causas, entendemos que há prevalência das doenças relacionas a Pressão Arterial, a Infecção Puerperal, e as relativas ao conjunto de doenças com a determinação no quadro de "Outras", essas por finalidade são as complicações que podem ocorrer na gravidez, descritas através; da ruptura de útero, placenta prévia, descolamento prematuro de placenta, gravidez tubária entre outras complicações relacionada diretamente aos órgãos da mulher e ao efeito que a gravidez produz sobre eles, na apreciação do quadro observamos também que a causa definida como "Choque" possui uma estabilidade de 1 caso por ano, tendo em 2012 uma única elevação para 3 casos/ano e em 2013 com 2 casos/ano, tornando a estabilidade de 1 caso/ano a parti de 2014.

Tabela 1 - Distribuição da Frequência de MMOD por CID 10 em Manaus no período de 2011 a 2015.

\begin{tabular}{|c|c|c|c|c|c|c|}
\hline \multicolumn{7}{|c|}{ Distribuição da Frequência de MMOD por CID 10 em Manaus no período de 2011 a 2015} \\
\hline Causas da MM & 2011 & 2012 & 2013 & 2014 & 2015 & Acumulado \\
\hline Infecção Puerperal & 6 & 1 & 0 & 3 & 0 & 10 \\
\hline Outras Infecções & 1 & 2 & 1 & 2 & 2 & 8 \\
\hline D. Recionadas a PA & 1 & 5 & 4 & 8 & 1 & 19 \\
\hline Choque & 1 & 3 & 2 & 1 & 1 & 8 \\
\hline Outras & 5 & 1 & 11 & 7 & 3 & 27 \\
\hline Total & 14 & 12 & 18 & 21 & 7 & 72 \\
\hline
\end{tabular}

Fonte: SEMSA, 2017. 
As denominadas "Outras Infecções" estão relacionadas com as infecções comunitárias, aquelas contraídas em seu meio de convivência comum, como a Infecção do Trato Urinário (ITU) e outra dessa natureza que agravadas colocam em risco o binômio mãe e filho. Em suma a grande maioria dos casos de MM ocorridas em Manaus são por causas obstétricas diretas e poderiam ser evitadas ainda na atenção básica, mais especificadamente no Pré-Natal.

Os dados relacionados ao Pré-Natal fornecidos pela SEMSA-MN, mostra no acumulado de 2011 a 2015 o exato número de 213.585 partos registrados na cidade e desses partos, 194.888 mulheres foram acompanhadas no Pré-Natal e 18.596 não acompanhadas, porém observou-se (Tabela 1) que o número de gestantes acompanhadas com mais de 7 consultas, ainda é insatisfatório, menos de $50 \%$ realizam o PréNatal de forma completa com no mínimo 7 consultas, esse número no programa de pré-natal tem grande relevância no estudos, pois muitas das etapas do Pré-Natal como exames realizados de acordo com o mês de gesta são negligenciados por muitas gestantes, nos levando a refletir quanto à qualidade da assistência, a capacidade dos profissionais, a estratégia utilizada para permanência da gestante ao programa, e a possibilidade de acompanhamento das gestantes frente suas necessidades básicas, entendendo que muitas trabalham e compõe renda familiar, quando não são as únicas provedoras da família.

Gráfico 1 - Distribuição da Frequência percentual de MMOD por CID 10 em Manaus de 2011 a 2015.

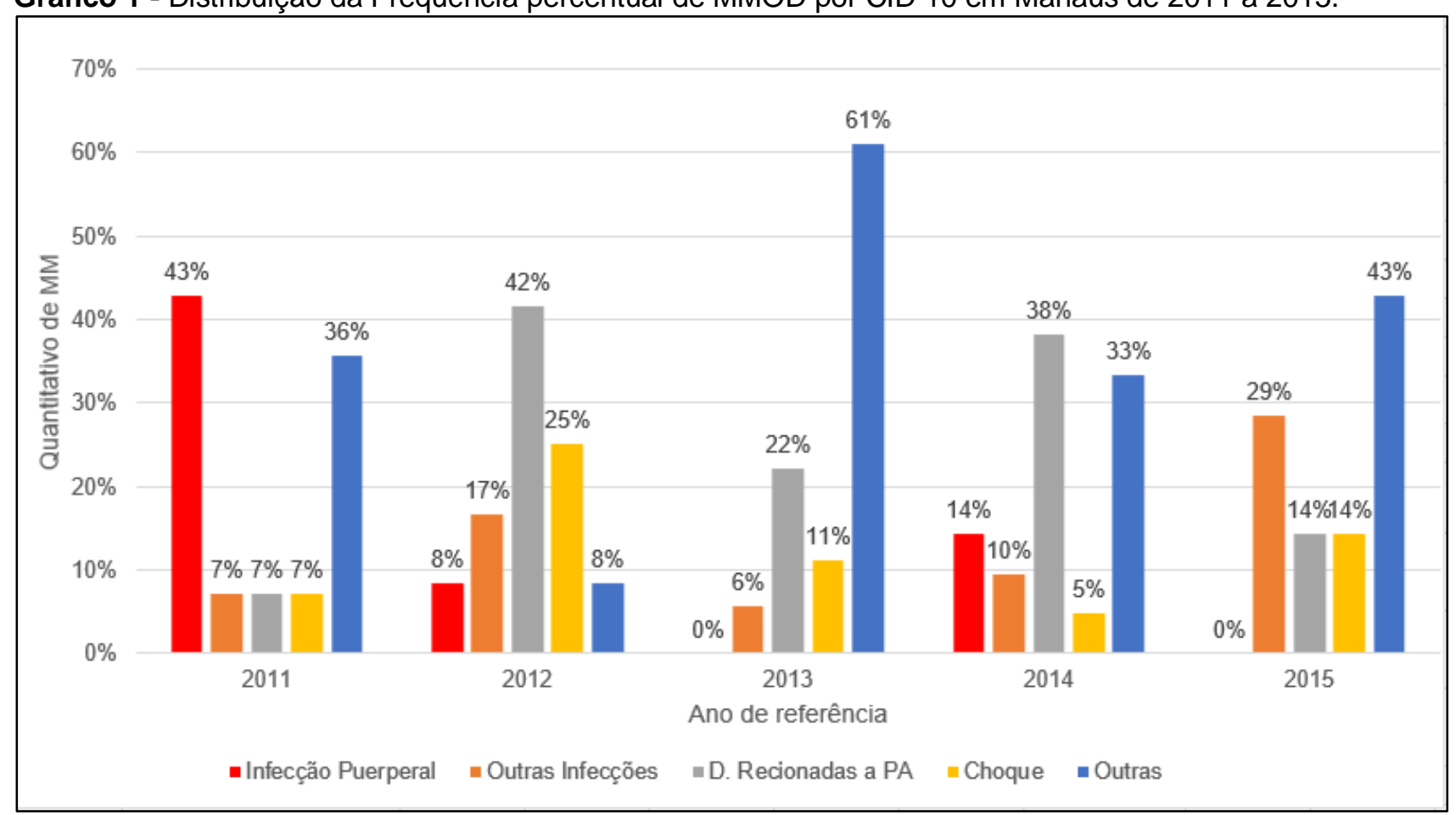

Fonte: SEMSA, 2017.

Após a análise gráfica, no gráfico 1, podemos apreciar os valores percentuais ano a ano, observamos que a infecção puerperal, vem caindo significativamente, porém as doenças relacionadas a PA tendem a um leve crescimento exponencial discreto, o choque, que está relacionado ao período cirúrgico ou em decorrência dele como: choque hipovolêmico, anafilático ou séptico, apresentou variações significativas entre os anos de 2012 e 2015, apresentando seu ponto máximo em 2012 com 25\% dos casos de MM e seu ponto mínimo em 2014 com 5\% dos casos, já o conjunto de anomalias na gravidez, determinada no gráfico como "Outras" teve um crescimento alarmante e pode estar relacionado a gravidez na adolescência, porém, se o Pré-Natal ocorresse de forma categórica conforme preconizado pelo Ministério da Saúde, a grande maioria dos casos poderiam ser evitados com o diagnóstico e tratamento precoce, se tratando das anomalias como gravidez ectópica, ruptura do útero, placenta prévia e descolamento prematura da placenta poderiam ser identificados em seus estágios iniciais com um simples exame de ultrassonografia. 
É relevante a observação dos dados que indicam desigualdades sociais, em especial os relacionados a escolaridade, pois segundo informações do Comitê Municipal de Prevenção do Óbito Materno e Infantil (CMPOMIF), o entendimento sobre prevenção, limpeza e autocuidado é melhor compreendido pelas famílias e gestantes com grau de ensino acima de 12 anos de escolaridade, nas fases gestacionais e no puerpério observamos que apenas $12 \%$ dessas mulheres veem a óbito, por essa razão entendemos que os casos de MM estão diretamente ligados ao conhecimento, devido ao entendimento sobre higiene, limpeza e necessidades. Porém vale ressaltar que ainda há muito casos de preenchimento inadequado das Declarações de Óbito (DO) nas zonas rurais da cidade de Manaus. Esses casos de preenchimento inadequado ou não preenchimento vem sendo combatido pelo comitê através de treinamento por Educação Continuada em loco e planos de ação voltados para excelência da assistência Pré-Natal e hospitalar, por meio de reuniões mensais e visitas aos postos de atendimento Pré-Natal e Hospitalares.

Em relação ao nível de escolaridade em anos de estudo das mulheres acometidas pela Morte Materna, consolidamos os dados fornecidos pela SEMSA-MN através do CMPOMIF e poderemos observar em números naturais e percentuais por ano e no acumulado do período, no Tabela 2 abaixo a referência de que $38 \%$ das mulheres encontravam-se entre o ensino fundamental ou no início do ensino médio, o que não determina uma faixa etária abaixo de 18 anos, pois em áreas periféricas da cidade ainda é comum esse retardo no ensino, Observamos também que $27 \%$ das mulheres acometidas pela MM haviam terminado o ensino primário, $12 \%$ terminará apenas a alfabetização, um ponto crítico encontrado na pesquisa foram os $5 \%$ de mulheres que não possuíam registro de escolaridade e os $2 \%$ nunca houvera estudo, Apenas $12 \%$ dessas mulheres acometidas pela MM possuíam nível escolar entre o ensino médio, superior ou além dele. Entendemos com essa análise que o nível de instrução/escolaridade afeta negativamente os conceitos do autocuidado em saúde, higiene pessoal e necessidades básicas para o bem-estar social, a exemplo podemos citar a noção de conservação ambiental e a percepção da necessidade de atuação da indivídua como cidadã em contextos sanitários coletivos, considerando em Manaus há muitas invasões com pouca urbanização.

Tabela 2 - Distribuição dos casos de MM por grau Instrução/Escolaridade Manaus de 2011 - 2015.

\begin{tabular}{|c|c|c|c|c|c|c|c|c|c|c|c|c|}
\hline \multicolumn{13}{|c|}{$\begin{array}{l}\text { Distribuição da Frequência de Morte Materna por Grau de Instrução/Escolaridade em Manaus/AM no período de } \\
\qquad 2011 \text { a } 2015\end{array}$} \\
\hline Ano de & \multicolumn{2}{|c|}{2011} & \multicolumn{2}{|c|}{2012} & \multicolumn{2}{|c|}{2013} & \multicolumn{2}{|c|}{2014} & \multicolumn{2}{|c|}{2015} & \multicolumn{2}{|c|}{ Total } \\
\hline Frequência & $\mathrm{N}$ & $\%$ & $\mathrm{~N}$ & $\%$ & $\mathrm{~N}$ & $\%$ & $\mathrm{~N}$ & $\%$ & $\mathrm{~N}$ & $\%$ & $\mathrm{~N}$ & $\%$ \\
\hline Não Informado & 2 & $10 \%$ & 2 & $9 \%$ & 0 & $0 \%$ & 2 & $5 \%$ & 1 & $5 \%$ & 7 & $5 \%$ \\
\hline Nenhum & 0 & $0 \%$ & 1 & $5 \%$ & 1 & $4 \%$ & 0 & $0 \%$ & 0 & $0 \%$ & 2 & $2 \%$ \\
\hline 1 à 3 anos & 5 & $25 \%$ & 1 & $5 \%$ & 1 & $4 \%$ & 5 & $12 \%$ & 4 & $20 \%$ & 16 & $12 \%$ \\
\hline 4 à 7 anos & 7 & $35 \%$ & 6 & $27 \%$ & 4 & $15 \%$ & 11 & $26 \%$ & 7 & $35 \%$ & 35 & $27 \%$ \\
\hline 08 à 11 anos & 3 & $15 \%$ & 10 & $45 \%$ & 14 & $52 \%$ & 18 & $43 \%$ & 5 & $25 \%$ & 50 & $38 \%$ \\
\hline 12 ou mais anos & 1 & $5 \%$ & 2 & $9 \%$ & 5 & $19 \%$ & 5 & $12 \%$ & 3 & $15 \%$ & 16 & $12 \%$ \\
\hline Ignorado & 2 & $10 \%$ & 0 & $0 \%$ & 2 & $7 \%$ & 1 & $2 \%$ & 0 & $0 \%$ & 5 & $4 \%$ \\
\hline Total de casos & \multicolumn{2}{|c|}{20} & \multicolumn{2}{|c|}{22} & \multicolumn{2}{|c|}{27} & \multicolumn{2}{|c|}{42} & \multicolumn{2}{|c|}{20} & \multicolumn{2}{|c|}{131} \\
\hline
\end{tabular}

Fonte: SIAB, DATASUS, Manaus.

Observando o gráfico 2 abaixo, podemos constatar que em Manaus a prevalência de mulheres atingidas pela Morte materna possuíam nível de escolaridade entre 8 e 11 anos de estudo, o que nos remete a entender que a grande maioria das mulheres vítimas da MM estão entre o final do ensino fundamental a meados do ensino médio, $27 \%$ das mulheres acometidas pela MM haviam terminado o ensino primário. Ao colocarmos em um avaliação mais ampla a relação grau de instrução/escolaridade, podemos observa que as mulheres que nunca estudaram, não possuem registros, estudaram apena o ensino primário e as que estudaram até o ensino fundamental ou início do ensino médio correspondem a $88 \%$ do total de mulheres acometidas pela morte materna, o que comprova a relação do autocuidado e grau de instrução/escolaridade.

REAS/EJCH | Vol.Sup.23 | e424 | DOI: https://doi.org/10.25248/reas.e424.2019 Página 6 de 12 
Gráfico 2 - Distribuição do Percentual de MM por grau Instrução/Escolaridade Manaus. 2011 - 2015.

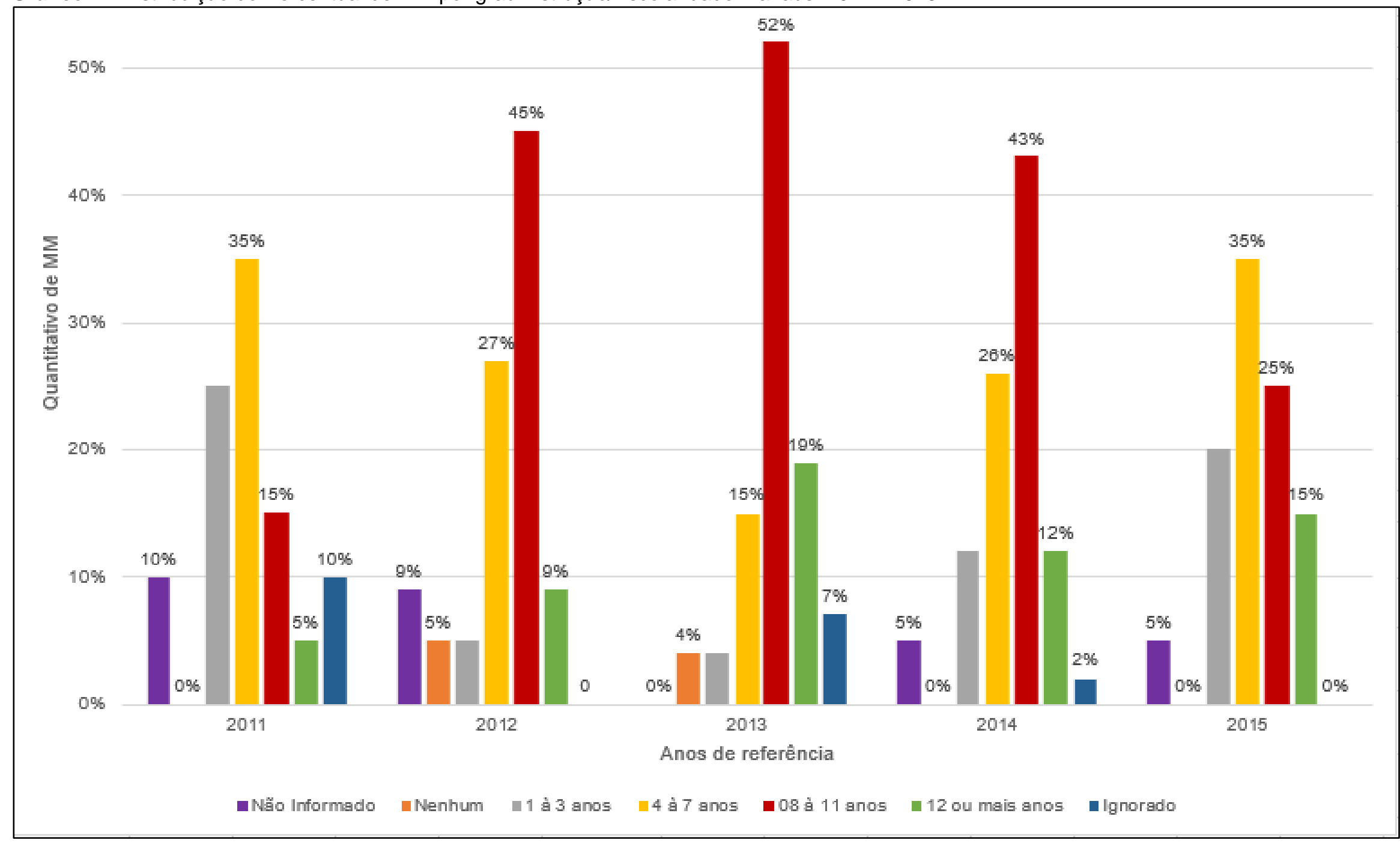

Fonte: SEMSA, 2017

REAS/EJCH | Vol.Sup.23 | e424 | DOI: https://doi.org/10.25248/reas.e424.2019 Página $\mathbf{7}$ de $\mathbf{1 2}$ 
A relação dos casos de Morte Materna entre as condições associadas, identificou-se iniquidades sociais relativas à escolaridade e raça com o predomínio de causas diretas de MM.

A análise feita no período de 2011 a 2015, identificou mulheres da raça parta com prevalência na evolução para Morte Materna, a raça pardas ocupou $79 \%$ desta população de mulheres acometidas com a MM, A população de mulheres caucasiano branca representam apenas $16 \%$, a população afro brasileira ocupou apenas $2 \%$ dos casos na cidade de Manaus, 3\% da população afetada por esse indicador não foram informadas, o que leva ao questionamento do preenchimento das Declaração de Nascidos Vivos (DNV), fato esse que vem sendo investigado pelo CMPOMIF, que já vem aplicando treinamentos e medidas para zerar esse indicador.

A população indígena e amarela não teve registros nessa série histórica, essas análises sobre a raça das mulheres acometidas pela MM, possuem condições que convergem com estes eventos na maioria das regiões brasileiras, esses percentuais variam de acordo com a composição de cor/raça predominante na região avaliada.

Frente a tal análise torna-se impreciso apontar a cor que apresenta maior risco em decorrência da intensa miscigenação racial na cidade de Manaus, este estudo observou a predominância da morte materna em mulheres pardas (79\%). Esta evidência pode estar relacionada a miscigenação racial local, mas há um ponto importante a ser apontado nesse fenômeno, muitas mulheres se definem como pardas e alguns profissionais quando possuem dúvidas na classificação racial/cor dessas mulheres durante o preenchimento da DNV e da DO, as classificam como de cor parda. É importante essa observação frente a esses dados, para realização de treinamentos relacionados ao preenchimento das Declaração de Óbito (DO) ou Declaração de Nascido Vivo (DNV), esses pontos já vêm sendo discutido pelo CMPOMIF e colocado como ponto fundamental nos treinamentos e reuniões do comitê.

Gráfico 3 - Distribuição do Percentual de Morte Materna por Raça em Manaus. 2011 - 2015.

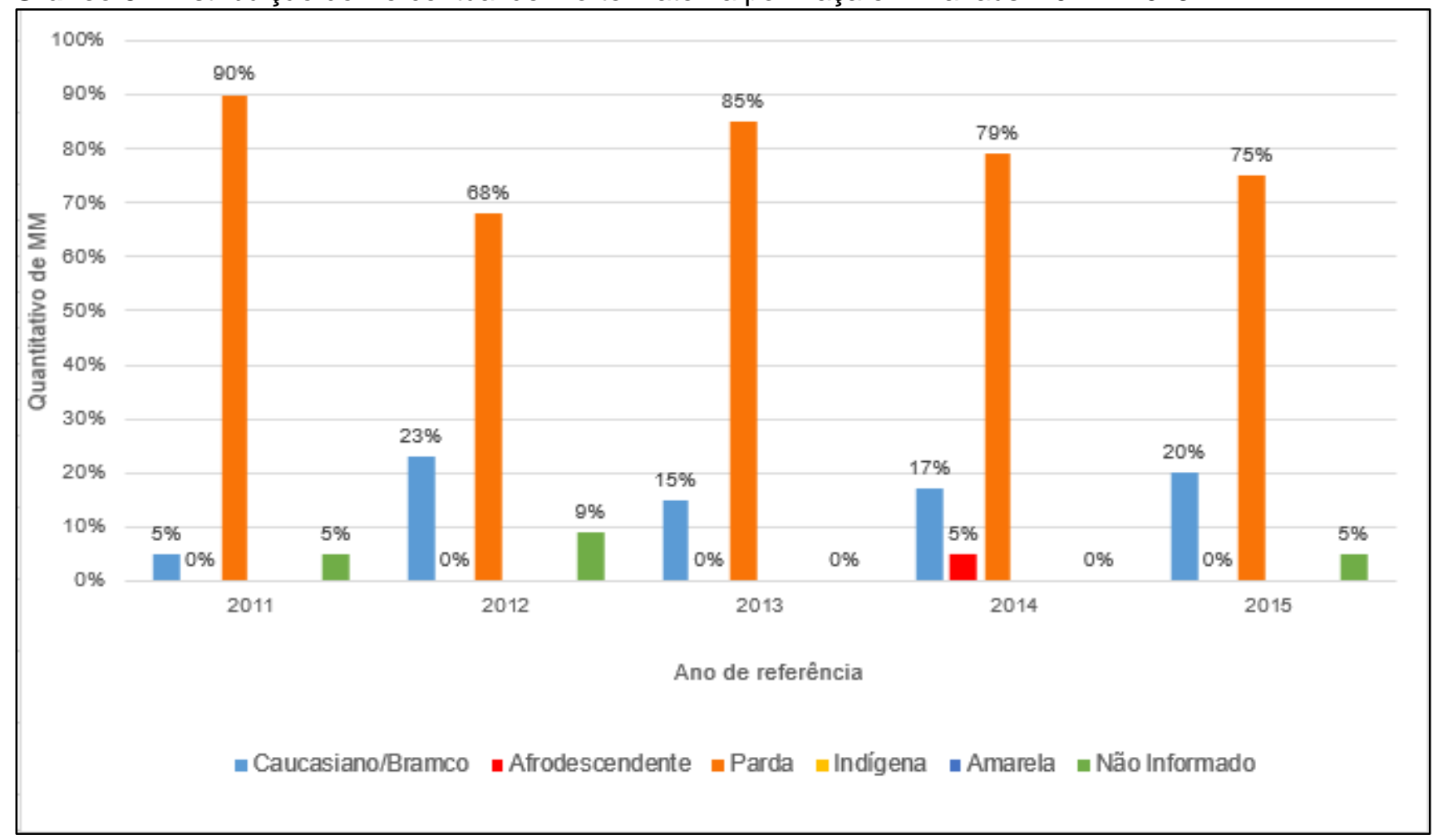

Fonte: SEMSA, 2017. 
Observando os gráficos 2 e 3 acima, podemos observar que em Manaus a prevalência mais atingida pela mortalidade materna respectivamente, é a raça parda e que o nível de escolaridade das mulheres atingidas por essa fatalidade concentra-se entre 8 e 11 anos de estudo, o que nos remete a entender que a grande maioria das mulheres vítimas da MM estão entre o final do ensino fundamental a meados do ensino médio. Frente a tal análise torna-se impreciso apontar a cor parda apresenta maior risco e esse predominância é decorrência da intensa miscigenação racial que a capital possui.

\section{DISCUSSÃO}

Em Manaus de janeiro de 2011 a dezembro de 2015, houve 131 casos de Morte Materna sendo 72 desses casos ou $55 \%$ relacionados a (MMOD), em segundo com 50 casos totalizando $38 \%$ do total de óbitos maternos está relacionado com a (MMOI), apenas 10 casos ou $8 \%$ desse total são classificados como (MMNE), o que sugere, que é necessário ampliação no processo de investigação. Os principais casos conforme o CID-10 estão relacionados no quadro 1 dessa pesquisa.

Segundo Ramos (2013), em seu estudo retrospectivo, apontou as principais causas de morte materna (MM), no Brasil, entre os anos de 2000 a 2009, foram por causas obstétricas diretas, com: (29\%) eclampsia e hipertensão gestacional; $(6,2 \%)$; hemorragia pós-parto $(5,9 \%)$; infecção puerperal $(5,2 \%)$; descolamento prematuro de placenta $(4,3 \%)$. Em estudo comparativo entre as regiões do país observou que na região Norte, a principal causa de morte foi por eclampsia (16,18\%). Um estudo sobre a análise de casos de mortes maternas, em Manaus, nos anos de 2001 a 2010, identificou 241 casos de MM, correspondendo a RMM de 63,2 / $100000 \mathrm{NV}$ e percentuais de causas direta e indireta de, respectivamente, 67,2 e $25,7 \%$, as causas de MM obstétricas diretas foram responsáveis por 162 casos e, as indiretas, por 62 casos. A maior proporção foi observada em mulheres com 4 a 7 anos de escolaridade (34,8\%), de raça não branca $(69,7 \%)$; com ocorrência predominante em hospitais públicos $(72,2 \%)$ de Manaus/AM. A evolução da RMM aconteceu em dois picos nos anos de 2003 e 2009. As causas de MM direta foram predominantes, com maior ocorrência nos anos de 2001 e 2006. No período de 2001 a 2010, os indicadores das MM de Manaus/AM reproduziram o panorama característico de países em desenvolvimento.

Fazendo uma análise quanto ao quantitativo de casos, classe e as causas de Morte Materna, observamos que proporcionalmente, houve mas casos neste estudo de série histórico de 5 anos, do que no estudo de Ramos acima citado, que avaliou 10 anos, no entanto, a classe de MMOD prevaleceu com percentual equivalente em nosso estudo que apresentou 72 casos (55\%), e, as causas com maior impacto na ocorrência dessas Morte Maternas mantiveram-se relacionadas a hipertensão gestacional, que em nosso estudo apresentou 19 casos, a infecção puerperal apresentou 10 casos, o choque 8 casos e os relacionados a outros tipos de patologias obstétrica diretas apresentou 27 casos registrados. Em relação as MM por causas indiretas, nosso estudo apresentou 50 casos (38\%), dentre eles se destacam, as doenças do aparelho respiratório e circulatório complicadas pela gestação. Outro ponto em comum dos estudos, é que a grande maioria dos casos ocorreu no ambiente hospitalar, independente do período gravídico a maioria no ambiente público, conforme os registros do CMPMIF.

A evolução da RMM em Manaus apresentou comportamento diferenciado estatisticamente significativo, ao longo do período de 2011 a 2015. Considerando os índices de 2011, onde a RMM era 47,25. Em 2012 apresentou valor de 50,61, pequeno aumento, em 2013 a (RMM) tem um crescimento significativo alcançado o patamar de 64,84. Mas em 2014 com RMM de 98,11 o valor mais que dobrou em relação ao de 2011. Já em 2015 o valor da RMM voltou ao patamar de 47,23. Valor esse pouco abaixo no evidenciado em 2011. A MMOD predominou em todo o período, com maior pico nos anos de 2013 e 2014 e menor ocorrência em 2011 e 2015, conforme dados do CMPMIF. Em relação ao percentual de MMOI, observa-se maior pico em 2014 e 2015. Em 2011 e 2013 foram registrados os menores percentuais de MM por causa indireta. Às maiores taxas de morte maternos concentram-se em 2014, o ano de 2015 expressa os menores índices de MM neste estudo. Fato este relacionado à implantação de medida e de controle do CMPOMIF.

REAS/EJCH | Vol.Sup.23 | e424 | DOI: https://doi.org/10.25248/reas.e424.2019 Página 9 de 12 
Em estudos similar, constatou-se que a baixa escolaridade correspondeu a 45,1\% das MM no período, de 2001 a 2010, equivalendo-se de resultados já relatados em grandes capitais do Brasil, como Recife e Rio de Janeiro, e outros. A escolaridade está fortemente associada a indicadores obstétricos negativos, que contribuem para elevação da ocorrência de MM em mulheres com baixo nível de instrução, muitos estudos brasileiros destacam a escolaridade como indicador das piores condições de vida e justiça social em país e reforça como grupo com maior risco para a mortalidade materna. (RAMOS, 2013). Em relação ao nível de escolaridade a pesquisa provou que, $38 \%$ das mulheres encontravam-se entre ensino fundamental ou o início do ensino médio, $27 \%$ haviam terminado o ensino primário, $12 \%$ terminará apenas a alfabetização, $5 \%$ não possuíam registro de escolaridade e $2 \%$ nunca houvera estudo, Apenas $12 \%$ dessas mulheres acometidas pela MM possuíam nível escolar entre o ensino médio, superior ou além.

A Morte Materna com maior prevalência nas bases inferiores da educação possui um entendimento simples, esse fenômeno se explica pela baixa escolaridade afetar negativamente a formulação de conceitos sobre os princípios do autocuidado em saúde. A relação entre o nível educacional e MM está ligada a noção de conservação ambiental e a percepção da necessidade de atuação da indivídua como cidadã em contextos sanitários individuais e coletivos, como; limpeza, saneamento descarte de lixo e higiene coletiva, residência e pessoal. (CASTRO; RAMOS, 2016).

Outra variável analisada nessa pesquisa no período de 2011 a 2015 foi a raça das mulheres que evoluíram para Morte Materna, sendo que as pardas são as mais acometidas na cidade de Manaus, ocupando $79 \%$ desta do grupo atingido, a população de mulheres caucasiana branca representam $16 \%$, a população afro brasileira ocupou apenas $2 \%$ dos casos, $3 \%$ da população afetada por esse indicador não foram informadas ou não possui registro na Declaração de Óbito, a população classificada como indígena e amarela não tiveram registro nessa série histórica, condição que converge com estes eventos na maioria das regiões brasileiras embora os percentuais variem de acordo com a composição de cor/raça predominante localmente. Uma importante observação frente a esses dados, é que em nossa região muitas pessoas se definem pardas e muitos profissionais ao preencher a Declaração de Óbito (DO) ou Declaração de Nascido Vivo (DNV), quando possuem algumas dúvidas quanto a raça, optam por registrar a cor parda.

Segundo Assayag (2015), esse dado pode ser compreendido pelo fato da maioria da população em Manaus ser classificada como parda, além do grande crescimento da população parda em todo o Amazonas.

Martins, (2004) relatou que em Manaus no ano de 2002 não houve casos de Morte Materna a mulheres Brancas, Mulheres negras e pardas tiveram 6 casos cada, as demais classificações de raça tiveram seus valores ignorados ou zerados, naquele ano houve 174.621 Nascidos vivos e a RMM ficou em 65,86. A autora levou em consideração que a morte materna está relacionada aos agravos que afetam mais a população negra em decorrência da evolução das doenças ou agravadas pelo tratamento ineficaz e dificultado pela falta de acesso aos serviços básicos de saúde ou falta do mesmo.

Conforme Ramos (2013), em seu estudo realizado em Manaus no período de 2001 a 2010, forma registrados 241 casos de morte materna sendo que esses acometeram 54 mulheres Brancas 22,4\%; Não Branca 168 casos 69,7\%; porém houve a situação de dados classificados como; não informado com 19 casos $7,9 \%$ do total. Esse estudo não estratificou de forma isolada os casos de MM nas raciais indígenas, negras e pardas, somando todas essas em uma única categoria denominada Não Branca, outro dado importante foi a quantidade de casos que não foram registrados na DO ou DNV a classificação da raça da mulher acometida pela MM.

Entendendo que a MM está relacionada com o baixo nível de escolaridade e atinge mais a raça predominante do local, muitos autores concordam que quanto maior o nível de conhecimento, menor é a razão da morte materna, quanto maior o investimento em educação e atenção básica menor é o resultado da RMM no Brasil, porém a MM com relação a escolaridade e raças possui predominância local alta em Manaus e mantém-se ainda em níveis elevados, com diferença entre as regiões. Os dados indicam desigualdades sociais, especialmente relacionadas à escolaridade e cor da pele. (CASTRO e RAMOS, 2016; BRASIL, 2009; Souza et al., 2008). 
Soares et al (2008), afirmam que na gestação, a mulher passa por profundas transformações, não só físicas como também emocionais. É importante conhecer alguns destes aspectos, pois alguns sintomas podem ser acentuados ou aliviados em função da capacidade de compreender a gestação e entender as próprias emoções.

Se tratando do risco da MM e concordando com todos os autores citados nessa pesquisa destacamos que o indicador de MM é um dos maiores problema da Secretaria de Saúde (SEMSA), assim como para sua população. Analisando todos os dados e informações adquiridas pelo CMPOMIF e demais estudos que dizem que entre 90 e $95 \%$ dos casos de MM poderiam ser evitados apenas com melhorias relacionadas às condições sociais e melhorias na qualidade da atenção básica, com a assistência à gestante durante o Pré-Natal, préparto, parto e puerpério.

Acreditamos que a pesquisa nos mostrou a Estratégia Saúde da Família (ESF) é responsável por garantir a adesão e a permanência das gestantes na rede de serviços relacionada a gestação, parto e puerpério. Quando não são alcançadas as metas da (ESF), muitas mulheres ficam expostas a situações de riscos, ou seja, à possibilidade para a ocorrência de óbitos materno, os programas já existem e são bem fundamentados pelo Ministério da Saúde, porém ainda falta muito investimento na melhorias sociais como; saneamento básico, educação e principalmente em saúde, no que se refere a atenção básica.

\section{CONCLUSÃO}

O resultado desta pesquisa nos mostrou que em Manaus ocorreram 136 casos de morte materna em mulheres na idade fértil, e destes casos, 131 foram relacionados a morte materna obstétrica, dividindo-se entre as suas classificações com; $55 \%$ de MMOD, 38\% de $\mathrm{MMOI}$, entretanto a prevalência das doenças relacionas a Pressão Arterial e a Infecção Puerperal, foram as causa que mais se repetiram dentro da classificação de MMOD, o quantitativo de partos não teve uma grande variação em seu total anual, porém esse valor está diretamente relacionado a RMM, que teve seu ponto máximo em 2014 com RMM de 98,11 e seus menores resultados permaneceram nos anos de 2011 e 2015, com média de 47,24 nesses dois anos. A pesquisa apontou ainda que $79 \%$ das mulheres acometidas pela morte maternas foram classificadas como pardas, quanto aos dados de escolaridade, observamos que a grande maioria das mulheres acometidas possuíam entre 8 a 11 anos de estudo, apresentando um percentual de $38 \%$; o grupo de mulheres com o nível de escolaridade entre 4 e 7 anos ocupou um percentual menor, de $27 \%$ do total de MM. É importante compreender a maioria da população feminina de Manaus é, ou se classifica como parda, devemos levar em consideração que essa classificação também é subjetiva ao profissional que preenche a DO. Entendemos os óbitos por causas obstétricas diretas podem ser evitados, mediante melhoria da assistência às mulheres no Pré-Natal, parto e puerpério, isso nos leva a refletir quanto à qualidade da assistência oferecida, no tocante ao Pré-Natal e as estratégias para manter as gestantes vinculadas ao programa, observando o cenário da desigualdade social e econômica ainda muito presente em Manaus, que só de intensifica quando agregado à complexidade geográfica presente no município, mesmo com toda a dificuldade a redução da RMM apresentou valores estatisticamente significativo, ao longo do período 2011 a 2015. Esses resultados reproduziram as características do município de Manaus, contudo, todas as medidas tomadas em prol da redução do indicador de MM e, sobretudo, as estratégias propostas pelo CMPOMIF deverão refletir de modo positivo no panorama de controle de MM em Manaus/AM, necessitando para isso, apenas de maiores investimentos na saúde básica por parte do Ministério da Saúde e Município.

\section{AGRADECIMENTOS E FINANCIAMENTO}

A SEMSA-MN por ter colaborado com o fornecimento de dados para a pesquisa através do Departamento de Informação, Controle e Regulação (DICAR). Ao Centro Universitário Luterano de Manaus - (CEULM/ ULBRA), Manaus - AM. Pela análise e aprovação do projeto em tempo hábil. Ao corpo docente da instituição, essenciais para o aprendizado e conclusão do mestrado em Saúde. 


\section{REFERÊNCIAS}

1. ASSAYAG Y. Mais da metade da população do Amazonas é jovem, parda, mostra PNAD. Jornal Em tempo, 13 de novembro de 2015. Disponível em: <http://d.emtempo.com.br/sem-categoria/48004/mais-da-metade-dapopulacao-do-amazonas-e-jovem-e-parda-mostra-pnad>. Acesso em 22 de outubro de 2017.

2. BRASIL. Portal Brasil. Mortalidade materna atingiu em 2011 menor índice dos últimos 10 anos, calcula Saúde. Portal Brasil. Publicado: 23/02/2012. Disponível em: http://www.brasil.gov.br/saude/2012/02/ministerio-dasaude-preve-que-2011-tera-reducaorecorde-da-mortalidade-materna. Acesso em 21 de outubro de 2016.

3. BRASIL; Ministério da Saúde; Conselho Federal de Medicina; Centro Brasileiro de Classificação de doenças. A declares de óbito: documento necessário e importante. 3ª ed. Brasília: Ministério da Saúde, 2009.

4. BRASIL; Governo do Estado do Ceará; Secretaria de Saúde - SESA/CE. Informe Epidemiológico - Mortalidade Materna de 09 de julho de 2015. Disponível em: file://C:/Users/Alexsandro/Downloads/informe_mortalidade_materna_junho_2015\%20(3).pd f. Acesso em: 16 de outubro de 2016.

5. CASTRO BMC, RAMOS SCS. PERFIL DE MORTALIDADE MATERNA EM UMA MATERNIDADE PÚBLICA DA CIDADE DE MANAUS-AM. Santa Maria, v. 42, n.1, p. 103-112, jan. /jun. 2016. Manaus-AM, 2016.

6. CASTRO BMC, MOYSÉS RPC, AMORIM MF. COBERTURA DE ASSISTÊNCIA AO PRÉ-NATAL E MORTALIDADE MATERNA: UMA ANÁLISE DE DADOS SECUNDÁRIOS NO MUNICÍPIO DE MANAUS-AM. Revista do Hospital Universitário Getúlio Vargas. v. 15, n. 1 (2016) / Manaus: Editora da Universidade Federal do Amazonas, 2016.

7. ONUBR, Nações Unidas no Brasil. A ONU e o desenvolvimento. Disponível em https://nacoesunidas.org/acao/desenvolvimento/. Com acesso em 01 de outubro de 2016.

8. PRESTES MLM. A pesquisa e a construção do conhecimento científico. 3. ed., 1. reimp. São Paulo: Rêspel, 2008.

9. RAMOS SCS. ANÁLISE DE CASOS DE MORTES MATERNAS EM MANAUS NOS ANOS DE 2001 a 2010. UFAM. Universidade Federal do Amazonas. Manaus-AM, 2013.

10. SEMSA; Secretaria Municipal de Manaus. Área Técnica de Saúde da Mulher. Disponível em: http://semsa.manaus.am.gov.br/. Acesso em 05 de outubro de 2016.

11. SEMSA-Mn, Secretaria Municipal de Saúde. SITUAÇÃO DE SAÚDE NO MUNICÍPIO DE MANAUS - Relatório Executivo. Manaus, 26 de abril de 2013.

12. SILVA RVG. Doença Hipertensiva Específica da Gestação - Projeto de Intervenção para o trabalho com as gestantes do território da Estratégia Saúde da Família no Município de Pedra da Anta - Minas Gerais. UFMG, MG 2015.

13. SOARES VMN, AZEVEDO EMM, WATANABE TL. Subnotificação da mortalidade materna no Estado do Paraná. Brasil: 1991-2005. Cad. Saúde Pública. Rio de Janeiro, 2008.

14. SOUZA KV, ALMEIDA MRCB, SOARES VMN. PERFIL DA MORTALIDADE MATERNA POR ABORTO NO PARANÁ: 2003-2005. Esc Anna Nery Rev Enferm, dezembro de 2008.

15. SOUZA JP. Mortalidade materna no Brasil: a necessidade de fortalecer os sistemas de saúde. Rev Bras Ginecol Obstet. 2011;

16. WINGERTER DG. ÓBITOS MATERNOS NO RIO GRANDE DO NORTE EM 2009 E A SUA EVITABILIDADE ANALISADA A PARTIR DA FERRAMENTA DE INVESTIGAÇÃO SIMWEB. XVIII Encontro Nacional de Estudos Populacionais, ABEP. Universidade Federal do Rio Grande do Norte - UFRN. Lindóia/SP - Brasil, novembro de 2012.

17. VIANA JFS. Óbitos Maternos por infecção Puerperal no período de 1998 a 2002 em Manaus - AM. UFAM Universidade Federal do Amazonas / FMT - Fundação de Medicina Tropical do Amazonas. Manaus - 2004. 